\title{
LA LÓGICA PERVERSA Y LAS EXIGENCIAS ACADÉMICAS. UNA APROXIMACIÓN A LAS PERSPECTIVAS DE LOS PROFESORES EN EDUCACIÓN FÍSICA DE LA UNIVERSIDAD NACIONAL DE LA PLATA (UNLP) SOBRE EL PROGRAMA DE INCENTIVOS A LOS DOCENTES- INVESTIGADORES
}

\section{MG. ALEJO LEVORATTI}

Profesor y Licenciado en Educación Física por la Universidad Nacional de La Plata (UNLP), Magíster en Antropología Social, IDES-IDAES-UNSAM. Estudiante del Doctorado en Ciencias Sociales y Humanas de la UNQ, Becario de la Comisión de Investigaciones Científicas de la Provincia de Buenos Aires, Investigador en Formación de la Universidad Nacional de Quilmes (UNQ) y Ayudante Diplomado en la Universidad Nacional de La Plata (UNLP) (Quilmes - Buenos Aires - Argentina)

E-mail: levoratti@gmail.com

\section{LIC. PAULA MACARIO}

Licenciado en Sociología por la Universidad Nacional de La Plata (UNLP). Doctoranda en Antropología Social, IDAES-UNSAM, Investigadora en Formación de la Universidad Nacional de Quilmes (UNQ) (La Plata - Buenos Aires - Argentina)

E-mail: paumacario@gmail.com

\section{RESUMEN}

Este artículo plantea como propósito profundizar en las perspectivas que los profesores en Educación Física de la UNLP, tienen sobre el Programa de Incentivos (PI). Para llevar a cabo este objetivo aborda, por un lado, al PI como política de Estado destinada a todas las disciplinas de las Universidades Nacionales, atendiendo a su capacidad de prescribir y determinar procedimientos y conductas. Por otro lado, analiza las lógicas propias de los actores destinatarios de aquél. El análisis posibilito dar cuenta de las percepciones y tensiones acerca del PI para los profesores en EF, al tiempo que este permitió en EF la aparición de un nuevo sujeto social el docente-investigador generando una serie de dificultades para su incorporación y crecimiento dentro de él.

PALABRAS CLAVES: Educación Física; configuración profesional; política universitaria, investigación. 
Entrevistadora: El Programa ya está en funcionamiento, es una política que lanzó el gobierno. Hay que ver qué hacen la Universidad, las Facultades y los distintos departamentos... Docente- Investigador: Yo te digo, Del Bello' no debe haber hecho más que el Programa de Incentivos, que no le sirvió ni a él. Pero la verdad es que generó una cantidad de cosas. Siendo un gobierno menemista, yo estoy en contra, pero aquél que quiso investigar pudo, cosa que antes no podía.

Entrevistadora: ¿Por qué no?

Docente- Investigador: Porque no había estructura. Qué podías hacer. Yo presentaba un paper en un congreso y quedaba ahí. Ahora me llaman de tal Universidad de acá o del extranjero porque estoy en el Programa de Incentivos. Hay un sistema que te permite, tiene sus errores, sus déficits, pero no es malo, es lo que se puede conseguir en la Argentina. (Extracto de entrevista realizada a un Docente-Investigador de la Carrera de Educación Física, en el año 2006)

\section{INTRODUCCIÓN}

Hace unos años atrás nos propusimos indagar en las prácticas de investigación en un grupo de profesores en educación física de la Facultad de Humanidades y Ciencias de la Educación de la Universidad Nacional de La Plata. En ese entonces, encontramos que los actores consideraban al Programa de Incentivos a los Docentes - Investigadores como una determinante significativa de la iniciación y la formalización de dichas prácticas, asociándolas con la configuración de su identidad. ${ }^{2}$ En esta oportunidad, nos planteamos como propósito profundizar en las perspectivas que este grupo académico tiene sobre esta política pública nacional destinada a las Universidades Nacionales. Nos interesa ahondar en la versión particular que este conjunto de actores construyó y construye sobre esta política pública, considerando los efectos que este Programa tuvo en sus perspectivas y prácticas en torno a

I. Del Bello fue secretario de la Secretaría de Políticas Universitaria (SPU) a partir del año | 993, secretaria creada ese mismo año.

2. En adelante nos referiremos a la Facultad de Humanidades y Ciencias de la Educación con las siglas FaHCE; Universidad Nacional de La Plata como UNLP; Educación Física como EF y al Programa de Incentivos a los Docentes - Investigadores como PI.

El Programa de Incentivos a los Docentes Investigadores es una política pública que fue formulada y puesta en práctica por el Poder Ejecutivo Nacional el 19 de noviembre de 1993. Tiene como objetivo central el desarrollo integrado de la carrera académica universitaria, promoviendo que las Universidades engloben actividades de docencia, investigación, extensión y gestión. Además plantea otros objetivos como el de incrementar las tareas de investigación y desarrollo a nivel nacional, la reconversión de la planta docente en las Universidades, el fomento de la mayor dedicación a la actividad universitaria y la creación de grupos de investigación.

Existen tres normativas del Programa de Incentivos a los Docentes - Investigadores. La primera está incluida en los Anexos del Decreto de creación 2427/93 del Poder Ejecutivo Nacional de 19 noviembre de 1993. La segunda denominada Manual de Procedimientos aprobado por Resolución Administrativa 665/ 97. Y una tercera que es una modificación del Manual de Procedimiento en el 2003. 
la investigación y su concepción de la educación física. Intentaremos mostrar los efectos ambiguos en torno al PI, sus críticas pero a la vez sus puntos destacados. El extracto de entrevista colocado al principio del trabajo a modo de epígrafe, condensa esta diversidad de puntos en tensión y relación que deben tenerse en cuenta para poder comprender las perspectivas que estos actores tienen sobre el PI. Allí encontramos, por un lado comentarios ideológicos y críticas a la normativa. Simultáneamente rescatan las posibilidades que generó y en particular para ellos, los profesores en EF, cuya disciplina no había podido desarrollar investigación de manera sistemática en una estructura que lo contenga y que la reconozca como una disciplina que investiga.

Para llevar a cabo este objetivo, el trabajo abordará el análisis desde un enfoque que comprenda, por un lado, al Programa de Incentivos, como política de Estado general destinada a todas las disciplinas de las Universidades Nacionales, atendiendo a su capacidad de prescribir y determinar procedimientos y conductas. Por otro lado, analizaremos las lógicas propias de los actores destinatarios de aquél. Para ello, atenderemos a la manera en que este grupo de docentes en educación física medió los efectos (apropiándosela, resistiéndola y/o resignificándola) de la aplicación de esta política de Estado. Esto implicará emprender la labor de forma holística, indagando en los distintos espacios en los cuales circulan y en la dimensión temporal-histórica en la cual se inscriben. Creemos que las formas específicas de sociabilidad del grupo académico en cuestión y sus relaciones sociales cotidianas, políticas, académicas y disciplinares, y la forma en cómo se articulan con la política más general, son claves para entender las condiciones que operan en el procesamiento de las políticas de Estado en el ámbito universitario y en la producción y reproducción de la vida social en las universidades.

Para realizar esta investigación se efectuaron en los años 2007 y 2010 entrevistas a docentes del Departamento de Educación Física quienes desempeñaban funciones docentes en el momento que se implementa el PI. Es pertinente aclarar que las mismas fueron realizadas, en su mayoría, a directores de proyectos y a docentes-investigadores categoría $\mathrm{V}$.

Al mismo tiempo nos servimos de publicaciones institucionales, reglamentos, resoluciones, revistas especializadas, libros, planes de estudio, programas de materias, documentos que fueron abordados e interpelados como espacio de expresión, significación y tensión de la perspectiva de estos actores.

El proceso de esta investigación se caracterizó por el trabajo sistemático y conjunto de estas diversas fuentes de información, procurando la construcción de datos y reconociendo los significados que los propios actores asignan a sus prácticas en sus contextos de manifestación. 
Este artículo se desarrolla a partir del diálogo con los estudios en educación superior (KROTSCH, 200 I), y en particular con aquellos que producen conocimiento acerca del PI. Marcelo Prati (2009) establece una clasificación de los estudios acerca del PI agrupándolos en tres posiciones que entrelazan consideraciones teóricas y empíricas con definiciones valorativas. Por un lado, agrupa a aquellos trabajos que ubica en un extremo crítico, representados principalmente por Paviglianiti, Marquina e Nosiglia ( 1996), Suasnábar (1999), Leal (2002). Tales trabajos señalan

[...] al Programa de Incentivos como parte de la agenda de política universitaria que el Banco Mundial y organismos análogos promovieron para América Latina en los 90, y que la Secretaría de Políticas Universitarias decidió implementar casi al pie de la letra [...] (PRATI, 2009, p. 46);

política que interfiere en la autonomía universitaria, a partir de mecanismos de control del trabajo académico (PRATI, 2009), exacerbando los componentes de individualismo, corporativismo y elitismo propios del trabajo académico (PAVIGLIANITI, MARQUINA E NOSIGLIA, 1996; SUASNÁBAR, 1995). Un segundo grupo de producciones, de carácter oficial, presenta al PI como parte de una trasformación positiva de la relación entre el Estado y las Universidades; como una estrategia que permite evaluar en el interior del sistema y de esta manera reconocer y premiar la excelencia. Por último, el autor señala una tercera posición que denomina intermedia, y en la cual él mismo se ubica, representada por Araujo (2002, 2003); Prati (200 I , 2002, 2009); Carullo y Vacarezza ( 1997); Fernández Berdaguer y Vaccarezza ( 1996); García de Fanelli (2005). Esta postura "[... ] reconoce ambivalencias en la implementación y efectos del programa” (PRATI, 2009, P. 47). El presente trabajo considera que tales estudios se han ocupado de manera aislada de diferentes dimensiones de la implementación y recepción del Pl; considerando de manera marginal los procesos de apropiación y mediación que los actores involucrados tienen de él. De todos modos, dialogaremos con sus planteos, dado que en muchas ocasiones sirven como punto de apoyo y comparación con las perspectivas de los actores bajo análisis en este estudio.

A continuación, en primer lugar destacaremos la situación previa a la ejecución del PI en torno a la investigación en EF de la FaHCE. En segundo lugar, señalamos las críticas que estos actores realizan al programa. En tercer lugar, destacamos algunas cuestiones significativas del proceso de categorización de los docentes y acreditación de proyectos entendiéndolos dentro de la lógica de estos profesores. Por último, en las consideraciones finales retomamos la idea de cómo este Programa, a pesar de la críticas y dificultades que planeta, ha incidido en la configuración de la identidad de al menos un sector de los profesores en EF. 


\section{PUNTO DE ARRANQUE DIFERENCIAL EN INVESTIGACIÓN}

Previo a la implementación del Programa de Incentivos, el desarrollo de las prácticas de investigación en los profesores en EF, que se desempeñan en la UNLP, era casi nulo. Si bien existieron "intentos de desarrollar proyectos de investigación" estos no prosperaron en el tiempo. No existían grupos que investigaran, los docentes no pertenecían al sistema institucionalizado de investigación nacional o provincial (CONICET, ANPCyT, CIC), no existían centros ni institutos de investigación, tampoco había formación de recursos humanos en investigación y la única experiencia de haber tenido un profesor con dedicación exclusiva no logró sostenerse. Según algunos entrevistados, la idea de investigar estaba latente aunque todavía no materializada: "Hubo algunos intentos, pero eran iniciativas de un grupo muy reducido que no siempre tenían un contexto o un marco institucional donde anclar". Fue el $\mathrm{Pl}$, así nos explicaban, el que generó "las exigencias académicas" para desarrollar las actividades de investigación. Cuando aparece este Programa quienes estaban a cargo de la dirección del departamento de EF decidieron aprovecharlo y comenzaron a generar las condiciones para cumplir con las exigencias que aquel establecía. Un integrante del departamento de ese entonces expresaba que había una necesidad de "orientar la disciplina de educación física hacia la investigación" y de "obtener dedicaciones mayores en la Facultad”. 3 Siendo el PI la estructura válida que se estaba implementando y teniendo en cuenta que la investigación se convirtió en la actividad de mayor prestigio y con reconocimiento económico, incorporarse a él era la forma para que sea reconocida como una disciplina que desarrolla investigación. ${ }^{4}$

El desarrollo de las prácticas de investigación de este grupo de profesores debe entenderse en un escenario más amplio. Las autoridades de ese momento de la FaHCE (Facultad en la que se encuentra el Departamento de EF) también consideraron al $\mathrm{PI}$ como una oportunidad para captar recursos económicos y para poder materializar las energías latentes que se encontraban en un sector importante de sus académicos, y llevar a cabo una activa y rápida estrategia. ${ }^{5}$ El resultado de la

3. Según Prati (2009), el Programa de Incentivos no sólo logró instalar la "cuestión" de la investigación en la agenda de un amplio conjunto de actores involucrados en los asuntos universitarios, sino que también logró dar un decidido impulso a una profesionalización académica con base en la investigación.

4. Para ampliar acerca de cómo incidió el PI en la estructura social y valorativa de las Universidades ver: Fernández Berdaguer y Vaccarezza (1996).

5. Debemos destacar que, como señala Prati (2009), uno de los efectos materiales que se logró en la Universidad Nacional de La Plata (UNLP) y en particular en la FaHCE a partir de la Implementación del PI (además del premio monetario y subsidios para los proyectos acreditados o para viajes) fue el incremento de dedicaciones exclusivas. La UNLP consiguió un refuerzo presupuestario de afectación específica para los participantes del Programa. Gracias a esta partida adicional, en 1994, la UNLP pudo ofrecer la extensión de su dedicación a muchos docentes, a condición de estar categorizados y participar en un proyecto de investigación acreditado. 
implementación de este Programa en la Facultad significó beneficios importantes en lo referente al aumento de dedicaciones exclusivas y en la sistematización e institucionalización de la actividad de investigación que se desarrollaba en esa unidad académica de una manera aislada, poco sistematizada y en muchos casos de manera vocacional.

El PI se encontró en la FaHCE con disciplinas con diferente desarrollo en investigación. Lo que implicó para ellas distintos esfuerzos de adaptación a un Programa que se presentaba homogéneo para todas y al que había que adaptarse. La situación en EF, en relación a la investigación, era mucho más débil y menos propagada en comparación a otras disciplinas de la FaHCE lo que significó un esfuerzo distinto, tanto en la incorporación al PI como en sostenerse dentro del él. ${ }^{6}$ Nos interesa destacar que son los propios actores los que dan cuenta del estado de poco avance en investigación que tenía la disciplina, caracterización que aparece reforzada al compararla con la situación de otros departamentos de la FaHCE.

Es importante mencionar que la formación de los profesores en educación física en el país estuvo orientada desde sus inicios a la preparación para el ejercicio de la docencia. Esta condición se debe en parte a que la educación se realiza principalmente en instituciones de formación docente de carácter terciarios, encontrándose actualmente en seis universidades nacionales la carrera de educación física, en donde las prácticas de investigación no tienen un rol preponderante.

Este punto de arranque diferencial en relación a la investigación creemos que es crucial para luego comprender las percepciones sobre el PI, sus críticas y la capacidad de respuesta a las exigencias y requisitos que éste planteaba y que actualmente plantea.

\section{ENTRE LA CRÍTICA Y LA ESTRUCTURA VÁLIDA}

Como dijimos anteriormente, el PI se presenta como un modelo homogéneo aplicado a realidades distintas, las cuales debieron acomodarse a dicho modelo. Según los estudios previos en torno al PI (ARAUJO, 2002; 2003), es posible afirmar que hay problemas y efectos no esperados del Programa, reconocibles en varias disciplinas de distintas unidades académicas, pero otros, lo sostenemos en este trabajo, son propios de la disciplina EF de la FaHCE - UNLP. A partir de, por un lado, lo que Araujo $(2002,2003)$ y Prati (2002) señalan acerca de la primacía que el PI le

6. Para ampliar sobre el proceso de implementación del PI en la FaHCE ver tesina de la Licenciatura en Sociología de la Lic. Paula Macario: Universidad, investigación: el Programa de Incentivos a los Docentes- Investigadores en la FaHCE- UNLP. 
asigna a los criterios de las ciencias duras y, por otro lado, Fernandez Berdaguer y Vaccarezza (1996) destacan que el PI privilegia como fuente de prestigio académico a la investigación -generando tensiones con el mérito de aquellos docentes cuyo carrera se funda en la docencia o en la actividad profesional-; las dificultades que se presentan en el campo de la disciplina de educación física para incorporase y sostenerse en el PI son varias.

Aquí acercaremos algunas reflexiones en torno a las perspectivas que los docentes en EF tienen acerca del PI como política general y como política que comenzó a ser palpable y parte de la labor académica cotidiana. Consideramos fundamental señalar que la investigación como práctica (nueva) en los profesores en EF no fue extensiva al conjunto de los profesores. ${ }^{7}$ A partir del análisis de los relatos de los actores hemos identificado dos grandes críticas al PI. Por un lado, críticas ideológicas, dado que surge en un contexto caracterizado como "neoliberal" y de grandes reformas al Sistema Educativo Argentino en general y de Educación Superior en particular. Por otro lado, críticas en relación a la normativa, evaluación y requisitos exigidos para ingresar y para sostenerse dentro del Programa.

El PI es una de las primeras de las varias políticas que implementa el gobierno argentino a través del Ministerio de Educación y otras instituciones creadas durante los primeros años de la década del noventa. La Secretaría de Políticas Universitarias, instituida en el año 1993, es una de las que ha tenido mayor incidencia en poner en marcha y dinamizar los programas en el sector universitario. El PI y el conjunto de políticas públicas destinadas a las Universidades se efectuaron en el periodo que se extiende de 1993 a 1997 que, según Pedro Krotsch (2002), corresponde a la "fase de construcción e implementación" de aquellas. Este contexto de transformaciones y reformas es caracterizado por varios investigadores como resultado de un cambio en la relación entre el Estado y las Universidades Nacionales (KROTSCH, 200 I, 2003; ARAUJO, 2003). Varios autores resaltan de este periodo la gran intromisión que el Poder Ejecutivo Nacional tuvo en las Universidades Nacionales a través de la formulación e implementación de estas políticas universitarias, identificadas como políticas sectoriales basadas en diagnósticos focalizados, dirigidas a solucionar problemas específicos, que en el año 1995 encuentran un marco general a partir de la puesta en marcha de la Ley de Educación Superior (PAVIGLIANITI; MARQUINA; NOSIGLIA, 1996; PAVIGLIANITI, 199I; KROTSCH, 200 I). Estos autores refieren a esta nueva relación entre Estado y Universidad como el pasaje del "Estado

7. Para ampliar sobre este tema ver el trabajo de Alejo Levoratti y Paula Macario (20 l 0) "Análisis de la configuración y actualización de las prácticas de investigación de los profesores en educación física de la Universidad Nacional de La Plata". 
Benevolente" al "Estado Evaluador", también como momento de "Recomposición neoconservadora". En este contexto, el PI fue criticado pero a la vez fue aceptado por la comunidad académica, lo que se traduce en el hecho estar dentro de la dinámica que el Programa propone.

En relación a la críticas ideológicas del PI, nos encontramos con los siguientes comentarios en los relatos del los docentes en EF. Un docente investigador con categoría IV nos dijo al respecto:

Yo soy muy crítico del PI. Creo que introdujo una lógica perversa adentro de la Universidad. Que es una manera de otorgar un sobresueldo encubierto, casi en negro, diría, por una vía que no es la correcta. Aquí hago una lectura ideológica clara: un modelo neoliberal lleva a una política de sobresueldos (...) me parece un mecanismo perverso. Es una forma de otorgar más plata a los docentes, pero por una vía que no es la correcta. Eso lleva a una lógica medio extraña. Quizás se investiga porque le gusta, pero quizás se investiga porque ahí hay un sueldo mayor.

A partir de la cita es posible identificar la referencia a la crítica del modelo neoliberal, y a la lógica perversa que genera la situación de que se paga un sobresueldo si se investiga. Es decir, que el docente investigador señala, por un lado, aquello que habilita el PI: investigar (aunque el riesgo es que quizás muchos hacen como si investigaran, aprendiendo a llenar planillas, para cobrar el sobresueldo), y por otro lado, y en línea con lo anterior, es que el Estado otorga más dinero a los docentes, pero a través de un gran intromisión de su parte, es decir a partir de la implementación de una normativa que estipula determinados requerimientos objetivos, y que de acuerdo al nivel del cumplimiento de esos requisitos por parte de los docentes depende la categoría equivalente de Investigación (CEI) y el monto del sobresueldo. ${ }^{8}$ Es decir, que reconoce que se le está dando más dinero a los docentes, pero a través de una lógica "perversa" y de "sobresueldo en negro".

Un docente- investigador, que fue Director del departamento, nos decía en relación a las prácticas de investigación que instauró el PI, "(...) como la palabra lo dice: incentivo a la investigación. Parece mentira que sea una tarea incentivada, no una tarea propia, pero bueno es la modalidad que hay".

Esta situación sobre la investigación, creemos que refuerza lo que se viene señalando: una intromisión del Estado en regular (y asignar dinero) a la práctica de

8. Para solicitar el Incentivo los docentes deben cumplir con una serie de requisitos y procedimientos: I. Haber sido categorizados, se les asigna una categoría equivalente de investigación (CEI), después de haber sido evaluados en sus antecedentes, en docencia, investigación, formación en recursos humanos, etc. 2. Participar en un proyecto de investigación acreditado por una entidad habilitada; 3. Dictar un mínimo de horas de clase frente alumnos al año (una parte importante en el grado). 
investigación si y sólo si se cumplen con los requisitos estipulados. Ahora bien, esos requisitos se pueden cumplir a partir de lo que Araujo $(2002,2003)$ llama "aprendizajes colaterales" que generó el PI que consisten en desarrollar mecanismos que permiten ingresar, permanecer y/o mejorar la posición en el sistema. ${ }^{9}$

(...) es un préstamo, que se devuelve con una tarea, en el otro es una función que es paga, ahí cambia totalmente la posibilidad de investigación, el tiempo del investigador, porque uno lo hace, y acá y hay que decirlo con todas las letras, debe mentir en las planillas el tiempo de investigación (...) porque necesita otros trabajos, por el incentivo propio no puede vivir, entonces es perverso.

La idea que se debe "mentir" para aparecer como si uno investigara es recurrente en los relatos de los docentes- investigadores, lo cual genera una lógica maligna. De todas maneras, están aquellos, como decía un docente más arriba, que investigan de "verdad".

El PI ha generado comentarios ambiguos es criticado pero a su vez es aceptado dado que muchos han ingresado al PI encontrando beneficios. En los relatos de los entrevistados aparece recurrentemente esa tensión que se expresa en una crítica al PI pero simultáneamente un comentario positivo al mismo.

En torno a las críticas en relación a los requisitos, percibimos, a partir de los relatos de los entrevistados, que éstas se centran en el sistema de evaluación y control del Programa. A continuación presentamos un conjunto de ejes con extractos de entrevistas que dan cuenta de esta tensión entre crítica y las condiciones que posibilitó:

a) El PI significó mayores tareas: "El Programa me atareó más pero me permitió mejorar la enseñanza".

b) Existen retrasos en el cobro del incentivo y demoras en las evaluaciones: "La gente se queja del PI, porque cobran tarde, porque los evalúan cuando quieren; la gente se queja, pero creo que en general te da prestigio; si ponés en el currículum que sos investigador del PI, la gente lo ve como positivo".

c) No hay docentes- investigadores I y II en la disciplina de Educación Física, por lo tanto tienen que ser evaluados por otras disciplinas: "(...) inclusive el problema más grande que tiene con nosotros es encontrar evaluador".

9. Araujo $(2002,2003)$ muestra efectos deseados y no deseados del PI, su "cara explícita" y su "cara oculta". Sostiene que los docentes investigadores han realizado dos tipos de aprendizajes como consecuencia de su participación en el PI. Por un lado, aprendizajes coherentes con los objetivos gubernamentales: participar de proyectos de investigación, publicar, realizar posgrados. Por otro lado, han incorporado "aprendizajes colaterales" no deseados: calcular sus acciones, a especular, a competir y desarrollar mecanismos que permiten ingresar, permanecer y/o mejorar la posición en el sistema. Incluso señala que han aparecido "conductas fraudulentas", como el autoplagio, la apropiación de ideas ajenas o falsa inclusión como coautor de publicaciones. 
d) En la planilla del PI Educación Física no aparece como disciplina que investiga: "No, ni como disciplina, ni como área, tenemos que poner 'otras' en la planilla winsip...".

e) Se publica porque se quiere divulgar conocimiento o por necesidad de tener certificado y publicaciones para sumar puntos: "Para estar en el PI y estar categorizado tengo que tener artículos publicados, ponencias. A mí una pregunta que me cabe permanentemente es: todos los que presentan tantas ponencias tiene realmente interés en divulgar los conocimientos, en divulgar lo que están investigando o lo hacen también solamente para obtener un certificado".

f) Los criterios de evaluación son poco claros, dispares y la normativa se ha interpretado rígidamente en determinadas situaciones: "Hay veces que a una persona le daban una categoría y a otra con antecedentes similares no se la daban. Evaluaciones desparejas, poco claras, criterios dispares, cosas que no terminan de cerrar".

g) El PI tiene una lógica tradicional de la ciencia, evalúa según producción: "El PI tiene una mirada tradicional de lo que es la investigación pero tiene el beneficio que te permite investigar como vos quieras, siempre y cuando cumplas con los requisitos de las planillas".

Este es un punteo de tensiones reconstruido a partir de las entrevistas. Como ya dijimos algunos problemas son extensibles a otras disciplinas. Existe otra tensión que es la dificultad de caracterizar en términos valorativos al PI. En general los comentarios en torno a si el Programa es bueno o malo surgen repetidamente: "El sistema tiene sus errores, sus déficit pero no es malo, es lo que se puede conseguir en la Argentina. Tiene una cierta sensibilidad que me maravilla que me parece mejor que el sistema mexicano y el brasilero".

Según con qué se compare o se evalué el PI, éste es bueno o malo, sirvió o no sirvió, permitió crecer u obstaculizó. Por ejemplo, los docentes- investigadores en EF al comparar la situación en relación a la investigación previa y posterior a la implementación del PI, afirman que antes en educación física no se investigaba y ahora sí. Y esto creemos ha tenido implicancias en la configuración de la identidad de esta disciplina y de los docentes en EF.

Si bien reconoce que EF dentro del PI tiene algunas desventajas, el propósito es generar nuevas condiciones hacia dentro del mismo: "Nosotros nos damos una política de crear nuestro lugar", "en algún momento vamos a tener nuestro propios evaluadores". El docente- investigador intenta relativizar los problemas y sostener la política que se dio en el departamento, dado que permite a quienes representa el campo de la EF en le FaHCE - UNLP disputar en términos legítimos un discurso teórico y práctico de lo que es la EF. 


\section{EL PROCESO DE CATEGORIZACIÓN, ACREDITACIÓN DE PROYECTOS: HACIA UNA NUEVA CONFIGURACIÓN DE LA IDENTIDAD}

Como se planteo arriba los docentes para poder solicitar el Incentivo deben cumplir una serie de requisitos y procedimientos. En este apartado, nos interesa profundizar y comprender, desde la perspectiva del sector de los profesores bajo la órbita de este estudio, las críticas al proceso de categorización y a los procedimientos de presentación y evaluación de proyectos. ${ }^{10}$ En estos procedimientos, en particular el primero, es en líneas generales, como dice Prati (2002, p. 196) "quizás el elemento de mayor impacto del Programa, a la vez que el más conflictivo". En el caso de EF, vemos que se presentan algunas características particulares en relación al momento de incorporación de los docentes al PI y la dificultad de obtener categorías altas (I y II), este último punto es de especial interés, dado que es donde se concentran las mayores críticas como ya fueron planteadas.

A continuación detallamos la cantidad de docentes en EF que se fueron categorizando en las distintas convocatorias," " en la del año 1994 se categorizaron doce docentes, en donde tres de ellos obtuvieron la categoría C y nueve de ellos la $D^{12}$. A partir de la implementación del Manual de Procedimientos del año 97 las categorías pasan a ser cinco: I, II, III, IV, V. En este caso son las categorías I, II y III'3 las que están habilitadas para dirigir proyectos de investigación acreditables. ${ }^{14}$ En el año 1998 se realizó un nuevo proceso de categorización donde se presentaron veintitrés docentes, de los cuales seis de ellos obtuvieron la III, cuatro la IV y trece la V. La CEl más elevada alcanzada por los Docentes del departamento de EF fue la C (posteriormente la III). A nivel nacional, no existía, al momento de realizar este trabajo, en la disciplina docentes- investigadores con CEl I ó II.

10. El proceso de categorización y el de acreditación de proyectos son procesos de evaluación. En el primer caso se evalúa a personas y en el segundo a proyectos.

1 I. Hasta el momento hubieron cuatro convocatorias a categorización. La última se realizó en el primer semestre de 2009. sólo contamos con los datos de las tres primeras categorizaciones: 1994, 1998, 2004. Los resultados de la convocatoria 2009 no entran en el análisis de este estudio dado que a la fecha de realización de este trabajo no se encontraban publicadas.

12. Según el Manual de Procedimientos en ese momento en vigencia: la categoría C correspondían a investigadores en la fase superior de su formación y la $\mathrm{D}$ a investigadores que se inician en la investigación

13. La introducción de la categoría III intentaría solucionar el problema de la escasez de directores. A pesar de su importancia, hay dos indicios que muestran que el nuevo Manual de Procedimiento le dio un tratamiento más formal que sustantivo: I. No se incluyo a la categoría III en el circuito nacional de categorización, 2. fue asimilada en el cobro a la CEI IV, con lo cual la ampliación de las facultades de esta categoría respecto de la anterior C no tiene contrapartida económica. Esto se va a modificar en el 2003. (ver PRATI, 2002)

14. "Si bien no se definen equivalencias automáticas entre las viejas y las nuevas categorías, la evolución posterior de los acontecimientos mostró que los A tendieron a ser I, los II, B, los D, V y los C se dividieron en dos grupos, accediendo unos a la III y otros a la IV" (PRATI, 2002: 208) 
El primer proyecto en la disciplina se denominó "Educación física: identidad y crisis", dirigido por una docente del Departamento de Ciencias de la Educación de la FaHCE y fue codirigido por el Director del Departamento de EF en el año 1993 , quien había obtenido la Categoría Equivalente de Investigación (CEI) C. La referencia a este primer proyecto como el puntapié inicial de las prácticas de investigación formalizadas es compartida por todos los docentes-investigadores entrevistados. Un actual profesor adjunto del departamento, que también lo era en ese momento, que no participó del mismo decía:

La génesis fue un equipo de investigación "Educación física: identidad y crisis". De ese equipo se fueron desprendiendo otros y se fue formando gente que fue generando a su vez otros equipos. Actualmente muchos de los que estaban en ese equipo hoy están dirigiendo otros. Fue una especie de ramificación. De tener uno solo hace diez años, ya estamos en los seis o siete equipos de investigación de Educación Física. Para mí fue una multiplicación, en no demasiado tiempo, bastante importante de cantidad de investigadores, de cantidad de equipos, de cantidad de temáticas.

La dirección de quien era en ese momento directora del departamento de Ciencias de la Educación, se debió a las exigencias de la normativa del PI que estipulaba una determinada categoría para desempeñar el rol de director.

A pesar de las distintas críticas que nos plantean los actores en torno al PI, éste sigue siendo la estructura válida que posibilitó la realización de prácticas de investigación.

Hemos señalado los inconvenientes que los docentes tuvieron y tienen para ingresar, permanecer y/o mejorar la posición en el Programa, pero es posible subrayar, aquello que fue tangencialmente apareciendo en las extractos de entrevistas mencionados arriba que creemos hace a un cambio sustantivo en la identidad (de los profesores y de la disciplina), que es que los profesores en EF (al menos un sector importante) pasan a ser docentes-investigadores y la disciplina EF, con una marcada tendencia hacia la docencia y enseñanza, pasa a ser una disciplina que investiga.

Los procesos de categorización y acreditación de proyectos de investigación, son destacados por los actores como las condiciones que generó el programa en esta disciplina, posibilitando la aparición dentro de este sector de docentes de un nuevo sujeto social: los docentes-investigadores, categoría que los posiciona en un lugar de paridad con las otras disciplinas. En este sentido, tomamos la propuesta de Mary Douglas (1996, p. 147), que al estudiar las formas de clasificaciones institucionales da cuenta de como éstas etiquetan "[...] y, hasta cierto punto, incluso crean las realidades a las que se aplican”, así es como "inventan a la gente”. Su planteo enfatiza que en la dinámica institucional "acaban por cambiarse los nombres y se vuelve a calibrar las 
gentes y las cosas para que encajen en las nuevas categorías" (DOUGLAS, 1996, p. I 59). El estudio de Douglas nos habilita a analizar que el PI, tiene un doble efecto beneficioso para EF: no sólo la ubica bajo el rótulo de disciplina que investiga sino que también los profesores pasan a ser identificados como docentes- investigadores. Esta etiqueta de docente- investigador los coloca en situación de par con las otras disciplinas de la Facultad y con el resto de los docentes- investigadores, habilitando nuevos diálogos. Estas nuevas interacciones y la significación del PI deben entenderse dentro de una tensión histórica que se daba entre la EF con las otras disciplinas de la FaHCE como plantea Alicia Villa (2003), ${ }^{15}$ por el reconocimiento en torno a otorgar o no rango de carrera a la EF, tal discusión está asociada al dominio de unas disciplinas "más serias" sobre otras, "lo cual devela la condición netamente política del debate en torno a la creación de cualquier carrera que exprese algún dominio de saber escasamente legitimado, como es el caso de la Educación Física" (VILLA, 2003).

Para este sector de profesores en EF, las prácticas en investigación en el marco del PI, no sólo les da la legitimidad institucional para sostener un discurso y posición acerca de lo que es la EF sino que también señalan cierta incidencia en la posibilidad de acceder a cargos político institucionales. Un ex Director nos dijo al respecto: "El reconocimiento de la carrera es que nosotros llegamos hasta la posición de Vicedecano, Secretario de Extensión, Consejeros Académicos, integramos lo que sería la parte ejecutiva y esto es importante, antes no, ni arrimábamos a eso" (LEVORATTI; MACARIO, 20I0).

En este sentido las críticas a los procesos de categorización de los docentes y a la acreditación y evaluación de los proyectos deben entenderse a partir de la perspectiva sobre el Programa y como éste se inscribe de forma particular para ellos.

\section{CONSIDERACIONES FINALES}

En este trabajo nos propusimos indagar en las perspectivas de un sector de los profesores en EF de la UNLP sobre el PI, teniendo como principio teórico-metodológico que para comprender el proceso de apropiación, críticas y resignificación de esta política pública se las debe analizar dentro de la trama de significación en que se encuentran los actores bajo estudio. En este sentido, sostenemos que

15. Villa (2003) al estudiar la historia del profesorado en Educación Física en la UNLP plantea que desde su creación existe una doble tensión de la posición de la carrera a partir de su incorporación a la Universidad. La primera, refiere a la tensión con las otras instituciones no universitarias y la formación universitaria, en donde la formación de los profesores de la UNLP por estar en la FaHCE se sustenta en la "humanidades" mientras las instituciones terciarias no universitarias sostienen un enfoque "anatomofisiológico, empírico, centrado en la salud física", o en términos de la clasificación arriba mencionada, en un enfoque vinculado con la corriente "físico- deportiva". La segunda tensión, interna a la Universidad, se da en torno a otorgar o no rango de carrera a la Educación Física" (2003). 
las críticas y/o las posibilidades que los docentes universitarios que participan del Programa de Incentivos detectan de él, deben ser analizados teniendo en cuenta sus trayectorias y las tradiciones y lógicas disciplinares e institucionales; de lo contrario, caeríamos en el presupuesto de que el conjunto los docentes universitarios se encuentran con las mismas posibilidades sin importar la disciplina e institución en la cual se inscriben; es decir homogeneizaríamos reacciones y efectos que fueron, en muchos casos, diversos e implicando diferentes esfuerzos para incorporarse y sostenerse dentro del Programa.

Asimismo nos resulta importante plantear la problemática de cómo el Programa de Incentivos, permitió dentro de una disciplina, EF, la aparición de un nuevo sujeto social, que son los docentes-investigadores, y, una nueva práctica sistematizada, la investigación; lo que re-configuró de diferentes maneras las relaciones de esto en la disciplina y la institución, imponiendo nuevas interacciones, que por cierto sobrepasan a la existencia del Programa. Nos referimos tanto a la relaciones intra FaHCE en cuanto a la legitimidad y rol de la EF con; y a las relaciones extra Facultad, con los institutos de formación en EF y otras Universidades para quienes la EF de la FaHCE de la UNLP se convirtió en un "punto de referencia de producción teórica". Desde el PI en adelante tomó impulso un procesos de desarrollo y materialización de actividades asociadas a las investigación (congresos nacionales e internacionales, publicaciones de libros y revistas, la creación de postgrados, investigaciones en la disciplina y la creación de la Licenciatura en Educación Física) que convierten a la EF de la UNLP en una disciplina que investiga.

\section{The Perverse Logic and Academic Demands: An Approach to the} Perspectives of Physical Education Teachers at the National University of La Plata (UNLP) on the Programa de Incentivos a los Docentes Investigadores

ABSTRACT: The proposal of this article is to analyze the perspectives of the teachers of Physical Education of the UNLP as regards the Programa de Incentivos (PI). On one hand, it analyses the Programa de Incentivos as a State policy directed to all National Universities, attending to its capacity in setting and determine actions. On the other hand, this article looks into understanding the dynamics characteristics of the actors/agents. This study tries to show the perspectives and tensions generated by the Pl, since at the same time it made possible the emergence of a new social actor, the teacher- researcher, as well as it generated many difficulties in its incorporation and development.

KEYWORDS: Physical Education; professional training; University policy; investigation. 
A lógica perversa e as exigências acadêmicas: uma aproximação às perspectivas dos professores de Educação Física da Universidad Nacional de La Plata (UNLP) sobre o Programa de Incentivos dos

\section{Docentes- Investigadores}

RESUMO: Este artigo propõe aprofundar as perspectivas que os professores de Educação Física da UNLP tem sobre o Programa de Incetivos (PI). Para realizar este objetivo aborda, por um lado, o Pl como política de Estado destinado a todas as disciplinas das Universidades Nacionais, atento à sua capacidade de prescrever e determinar procedimentos e condutas, e por outro lado, analisar as lógicas dos próprios atores receptores. O estudo possibilitou observar as percepções e tensões geradas pelo Pl, pois enquanto na EF permitiu o surgimento de um novo sujeito social, o docente-investigador, também gerou muitas dificuldades para sua incorporação e crescimento dentro dele.

PALAVRAS CHAVES: Educação Física, configuração profissional, politica universitária, pesquisa.

\section{REFERENCIAS}

ARAUJO, S. M. Universidad, investigación e Incentivos: la cara oscura. La Plata: Ediciones al Margen, 2003.

ARAUJO, S. M. Evaluación, Incentivos a la actividad investigadora y trabajo académico. Algunas conclusiones en el estudio de un caso en la Argentina. In: KROTSCH, P (Org.). La Universidad cautiva: legados, marcas y horizontes, La Plata: Ediciones al Margen, 2002. p. 23 I - 25 I.

ATAIRO, D. Trama sociopolítica del gobierno universitario: análisis del funcionamiento de los órganos de gobierno a partir de la implementación de una política universitario. Fundamentos en Humanidades, Saint Luis. San Luis, v. 9, p. 85-1 10, 2008.

CARULLO, J. C.; VACCAREZZA, L. El incentivo a la investigación universitaria como instrumento de promoción y gestión de la I + D. REDES. Revista de Estudios Sociales de la Ciencia. Buenos Aires, v. 4, n. 10, p. I55- I78, oct., 1997.

DOUGLAS, M. Cómo piensan las instituciones. Madrid: Alianza, 1996.

FERNANDEZ BERDAGUER, M. L.; VACCAREZZA, L. S. Estructura social y conflicto en la comunidad científica universitaria: la aplicación del Programa de Incentivos para Investigadores Docentes en las universidades argentinas. In: ALBORNOZ, M. (ed.) Ciencia y sociedad en América Latina. Quilmes: Ed. de la Universidad Nacional, 1996. p. 250-264.

GARCIA FANELLI, A. Universidade, organización e incentivo; desafíos de la financiamiento frente a la complejidad institucional. Buenos Aires: Fundacion OSDE, 2005.

KROTSCH, P. Educación superior y reformas comparadas. Quilmes: Ed. da Universidad Nacional, 200I. 
KROTSCH, P. El Proceso de Formación e Implementación de las políticas de Evaluación de la Calidad en la Argentina. In: KROTSCH, P (Org.), La universidad cautiva: legados, marcas y horizontes. La Plata: Ed. al Margen. 2002. p. I53- 178.

LEVORATTI, A.; MACARIO, P. Análisis de la configuración y actualización de las prácticas de investigación de los profesores en educación física de la Universidad Nacional de la Plata. In: FREDERIC, S.; GRACIANO, O.; SOPRANO, G. (Coord.). El Estado argentino y las profesiones liberales, académicas y armadas. Rosario: Prohistoria, 2010. p. 533-566.

MARQUINA, M.; NOSIGLIA, M. C. Políticas universitarias en la Argentina 1983 - 1995: el papel del poder ejecutivo y del poder legislativo de la nación. Revista del Instituto de Investigaciones en Ciencias de la Educación, Buenos Aires, v. 4, n.7, p. 47-57, 1995.

PAVIGLIANITI, N. Neoconservadurismo y educación. un debate silenciado en la Argentina del 90. Buenos Aires: Libros del Quirquincho, 1991.

PAVIGLIANITI, N; MARQUINA, M-; NOSIGLIA, M. C. Recomposición neoconservadora. lugar afectado: la universidad. Buenos Aires: Instituto de investigaciones en Ciencias de la Educación: Universidad de Buenos Aires. 1996.

PRATI, M. D. El programa de incentivos como política pública: categorías para su análisis. In: 2das Jornadas de Sociología de la UNLP, Departamento de Sociología, FaHCE, UNLP. La Plata, 200 I.

PRATI, M. D. El Programa de Incentivos a los Docentes Investigadores: formulación, implementación y visiones sobre su impacto. In: KROTSCH, P (Org.). La Universidad cautiva: legados, marcas y horizontes, La Plata, Ediciones al Margen. 2002. p. 193-230.

PRATI, M. D. El Programa de incentivos y la "sociedad" universitaria. Revista Pensamiento Universitario, Buenos Aires, ano 12, n. 12, oct. de 2009.

SUASNABAR, C. Situación del sector universitario y las transformaciones del mercado académico. Revista Propuesta Educativa, Buenos Aires, v. 6, n. 13, p.97-105, 1995.

VILLA, A. La tradición humanista en la formación de profesores/as (Amavet y el caso del Profesorado en educación física. UNLP). Lecturas, Educación Física y Deporte: revista digital. Buenas Aires, n. 8, n. 56. enero, 2003. Disponível em: http://www.efdeportes.com/efd56/ amavet.htm. Accesso em: 13 de mar. de 2012.

Recebido em: 3 I out. 20 I I Aprovado em: 3 jul. 2012 
Endereço para correspondência:

\author{
Alejo Levoratti \\ Av. Paseo Colon No 1019 Piso I 8 Dpto "G" \\ CP: CI063ACK \\ Ciudad Autónoma de Buenos Aires.
}

Argentina.

Paula Lucia Macario:

Calle Estados Unidos 548 Dpto 3

CP:CI IOIAAL

Ciudad Autónoma de Buenos Aires

Argentina. 